\title{
The Cocaine and Oxycodone Biobanks, Two Repositories from Genetically Diverse and Behaviorally Characterized Rats for the Study of Addiction
}

(ㄴ) Lieselot L. G. Carrette, ${ }^{1,3}$ Giordano de Guglielmo, ${ }^{1}$-Marsida Kallupi, ${ }^{1}$ Lisa Maturin, ${ }^{1}$ Molly Brennan, ${ }^{1}$ Brent Boomhower, ${ }^{1}$ Dana Conlisk, ${ }^{2}$ Sharona Sedighim, ${ }^{1}$ Lani Tieu, ${ }^{1}$ McKenzie J. Fannon, ${ }^{1}$ Nathan Velarde, ${ }^{1}$ Angelica R Martinez, ${ }^{1}$ Jenni Kononoff, ${ }^{2}$ CAdam Kimbrough, ${ }^{1}$ Sierra Simpson, ${ }^{1}$ Lauren C. Smith, ${ }^{1,2}$ Kokila Shankar, ${ }^{1,2}$ Francisco J. Ramirez, ${ }^{1}$ Apurva S. Chitre, ${ }^{1}$ Bonnie Lin, ${ }^{1}$ Oksana Polesskaya, ${ }^{1}$ Leah C. Solberg Woods, ${ }^{4}{ }^{-}$Abraham A. Palmer, ${ }^{1,5}$ and ${ }^{\circledR}$ Olivier George ${ }^{1}$

\section{https://doi.org/10.1523/ENEURO.0033-21.2021}

${ }^{1}$ Department of Psychiatry, University of California, San Diego, La Jolla, CA 92093, ${ }^{2}$ Department of Neuroscience, The Scripps Research Institute, La Jolla, CA 92037, ${ }^{3}$ Center for Medical Genetics Ghent, Ghent University, Ghent 9000 , Belgium, ${ }^{4}$ Department of Internal Medicine, Section on Molecular Medicine, Wake Forest University School of Medicine,

Winston-Salem, NC 27157, and ${ }^{5}$ Institute for Genomic Medicine, University of California, San Diego, La Jolla, CA 92093

\section{Visual Abstract}

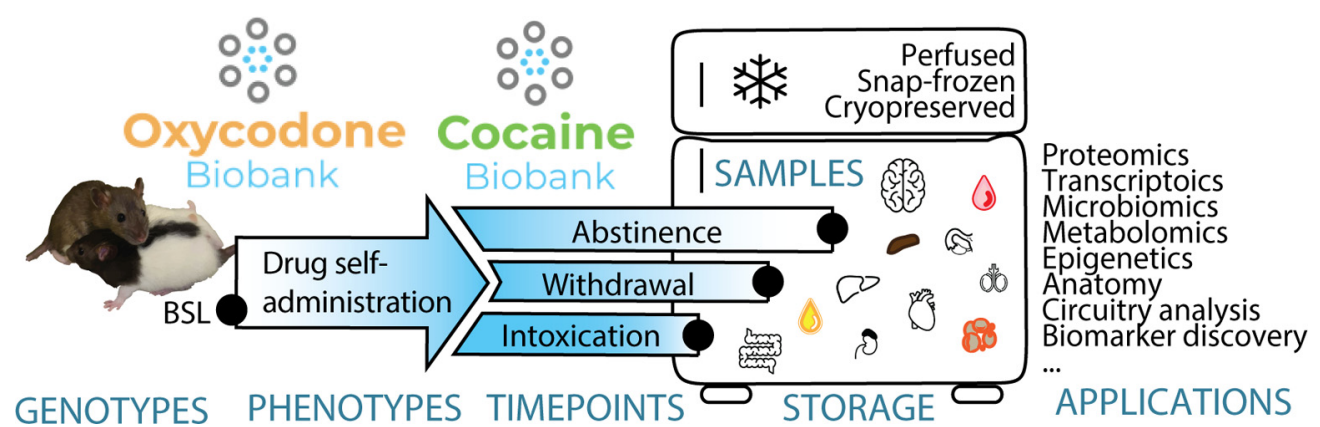

The rat oxycodone and cocaine biobanks contain samples that vary by genotypes (by using genetically diverse genotyped HS rats), phenotypes (by measuring addiction-like behaviors in an advanced SA model), timepoints (samples are collected longitudinally before, during, and after SA, and terminally at three different timepoints in the addiction cycle: intoxication, withdrawal, and abstinence or without exposure to drugs through age-matched naive rats), samples collected (organs, cells, biofluids, feces), preservation (paraformaldehyde-fixed, snap-frozen, or cryopreserved) and application (proteomics, transcriptomics, microbiomics, metabolomics, epigenetics, anatomy, circuitry analysis, biomarker discovery, etc.

\section{Significance Statement}

The cocaine and oxycodone biobanks offer genetically and behaviorally characterized, longitudinally, and cross-sectionally diverse samples to researchers free of charge. Through this resource, we want to make these valuable samples available to researchers who may not have the equipment or expertise to perform behavioral experiments but can make valuable contributions to the addiction field and facilitate the identification of biomarkers and the development of novel, effective therapies for substance use disorders (SUDs). 
Substance use disorders (SUDs) are pervasive in our society and have substantial personal and socioeconomical costs. A critical hurdle in identifying biomarkers and novel targets for medication development is the lack of resources for obtaining biological samples with a detailed behavioral characterization of SUD. Moreover, it is nearly impossible to find longitudinal samples. As part of two ongoing large-scale behavioral genetic studies in heterogeneous stock (HS) rats, we have created two preclinical biobanks using well-validated long access (LgA) models of intravenous cocaine and oxycodone self-administration (SA) and comprehensive characterization of addiction-related behaviors. The genetic diversity in HS rats mimics diversity in the human population and includes individuals that are vulnerable or resilient to compulsive-like responding for cocaine or oxycodone. Longitudinal samples are collected throughout the experiment, before exposure to the drug, during intoxication, acute withdrawal, and protracted abstinence, and include naive, age-matched controls. Samples include, but are not limited to, blood plasma, feces and urine, whole brains, brain slices and punches, kidney, liver, spleen, ovary, testis, and adrenal glands. Three preservation methods (fixed in formaldehyde, snap-frozen, or cryopreserved) are used to facilitate diverse downstream applications such as proteomics, metabolomics, transcriptomics, epigenomics, microbiomics, neuroanatomy, biomarker discovery, and other cellular and molecular approaches. To date, $>20,000$ samples have been collected from over 1000 unique animals and made available free of charge to non-profit institutions through https://www.cocainebiobank.org/ and https:// www.oxycodonebiobank.org/.

Key words: psychostimulant; opioid; substance-related disorders; biological specimen banks; outbred strains

\section{Introduction}

Substance use disorders (SUDs) are pervasive in society (Grant et al., 2016; Vadivelu et al., 2018), with dramatic societal and individual consequences. There are currently no FDA-approved pharmacological treatments for cocaine use disorder. While there are FDA-approved treatments for opioid use disorder, the efficacy of these medications varies, and the relapse rate remains high (Eap et al., 2002; de Cid et al., 2008). To improve current treatments and discover new ones, the individual differences in both the propensity to develop addiction-like behaviors (Vowles et al., 2015) and the response to treatment need to be better understood. A critical hurdle in the effort to identify biomarkers and targets is the limited number of repositories that include longitudinal samples, with a detailed characterization of SUD.

Received January 25, 2021; accepted March 31, 2021; First published April $15,2021$.

The authors declare no competing financial interests.

Author contributions: G.d.G., M.K., A.A.P., and O.G. designed research; L.L.G.C., G.d.G., M.K., L.M., M.B., B.B., D.C., S.Se., L.T., A.R.M., M.J.F., N.V., J.K., A.K., S.Si., L.C.S., K.S., F.J.R., A.S.C., B.L., and O.P. performed research; L.C.S.W. contributed unpublished reagents/analytic tools; L.L.G.C., G.d.G., M.K., and O.G. analyzed data; L.L.G.C. and O.G. wrote the paper.

The generation of the addiction biobanks was supported by National Institute on Drug Abuse Grants U01DA043799 and U01DA043799. L.L.G.C. was supported by a postdoctoral fellowship from the National Research FundFlanders. O.P. and A.A.P. were also supported by the National Institute on Drug Abuse Grant P50DA037844.

Acknowledgements: The George lab acknowledges the Preclinical Addiction Research Consortium at UCSD and thanks all student interns and volunteers who have helped out in this experiment.

Correspondence should be addressed to Olivier George at olgeorge@ health.ucsd.edu.

https://doi.org/10.1523/ENEURO.0033-21.2021

Copyright (C) 2021 Carrette et al.

This is an open-access article distributed under the terms of the Creative Commons Attribution 4.0 International license, which permits unrestricted use, distribution and reproduction in any medium provided that the original work is properly attributed.
To address this, we have created the cocaine biobank (https://www.cocainebiobank.org/) and the oxycodone biobank (https://www.oxycodonebiobank.org/). These repositories contain $>20,000$ samples from $\sim 1000$ individual rats. Indeed, numerous biological markers have been identified in preclinical models. Failure to replicate or translate such markers to human in the past could be linked to low sample size (usually $N=6-12$ ), lack of standard operating protocols between laboratories, and use of inbred strains. Every individual of $\sim 1000$ rats in the biobanks is fully characterized for addiction-like behaviors in a strictly controlled environment using state-of-the-art intravenous self-administration (SA) models with high relevance to SUD. The rats are from the heterogeneous stock (HS). These are highly recombinant animals, established by crossbreeding eight genetically diverse founder strains (Hansen and Spuhler, 1984; STAR Consortium et al., 2008; Baud, 2013; Solberg Woods and Palmer, 2019), resulting in a diversity that mimics the diversity found in the human population. Together with the behavioral characterization, this allows for genome-wide association studies (GWAS) for a variety of addiction-related quantitative traits (Valdar et al., 2006; Baud, 2013; Keele, 2018; Chitre et al., 2020) by whole-genome sequencing of each individual.

Throughout behavioral experiments, samples (feces, urine, blood) are taken longitudinally. Terminal samples (brain, heart, kidneys, liver, colon, ovaries or testis, adrenal glands, and peripheral blood mononuclear cells (PBMCs) are collected from different animals at three timepoints, during (1) intoxication, (2) acute withdrawal, (3) protracted abstinence or from naive rats. To maximize compatibility with a range of downstream applications, samples are (1) perfused using paraformaldehyde, (2) snap-frozen, or (3) cryopreserved. Since all rats are genetically and behaviorally characterized, samples from individuals with specific genotypes or phenotypes can be requested.

The biobanks are cost-effective for the scientific community and created with an open-source mindset (White 
et al., 2019). Samples are freely available to non-profit organizations. Genetic and behavioral data will be deposited in a public repository like the rat genome database (Smith et al., 2020) or Gene Network (Sloan et al., 2016). Protocols are disclosed here and online (https://www. protocols.io/workspaces/george-lab). The addiction biobanks are particularly useful for researchers from fields outside of addiction, who do not have the resources or expertise to perform chronic intravenous SA. We here introduce the biobanks with methodological details and an example of their application.

\section{Materials and Methods}

Detailed behavioral procedures can be found in the paper's extended information and the George lab protocol repository on protocols.io (https://www.protocols.io/ workspaces/george-lab). All of the procedures were conducted in strict adherence to the National Institutes of Health Guide for the Care and Use of Laboratory Animals and were approved by the Institutional Animal Care and Use Committees of The Scripps Research Institute and University of California San Diego.

\section{Animals}

$\mathrm{HS}$ rats (sometimes referred to as $\mathrm{N} / \mathrm{NIH}$ ) are provided by Leah Solberg Woods (the only colony in North America). The HS colony was transferred from the Medical College of Wisconsin to the Wake Forest University School of Medicine in 2016. The animals in the current study come from the Wake Forest University School of Medicine colony, which has been named NMcwiWFsm:HS (Rat Genome Database number 13673907). To minimize inbreeding and control genetic drift, the HS rat colony consists of $>64$ breeder pairs and is maintained using a breeding strategy that takes into account the kinship coefficient of the breeders (Solberg Woods and Palmer, 2019). To maximize genetic diversity, each breeder pair contributes only one male and one female to subsequent cocaine and oxycodone cohorts. The goal is to include 1000 rats in both biobanks consisting of half males, half females. Each rat receives a chip with a radiofrequency identification (RFID) code to keep track of them, their breeding, behavior, organs, and genomic info. Rats are shipped at three to four weeks of age, kept in quarantine for two weeks, and then for the rest of the experiment housed two per cage on a 12/12 h light/dark cycle (lights off at 8:00 A.M. for cocaine, lights off at 4:00 P.M. for oxycodone) in a temperature $\left(20-22^{\circ} \mathrm{C}\right)$ and humidity (45-55\%) controlled vivarium with ad libitum access to tap water and food pellets (PJ Noyes Company).

\section{Surgery for jugular vein catheterization}

Rats are anesthetized with vaporized isoflurane (1-5\%). Intravenous catheters are aseptically inserted into the right jugular vein. Catheters consist of Micro-Renathane tubing $(18 \mathrm{~cm}, 0.023$-inch inner diameter, 0.037-inch outer diameter; Braintree Scientific) attached to a $90^{\circ}$ angle bend guide cannula (Plastics One), embedded in dental acrylic, and anchored with mesh (1 $\mathrm{mm}$ thick, 2-cm diameter). Tubing is inserted into the vein following a needle puncture $(22 \mathrm{G})$ and secured with a suture. The guide cannula is punctured through a small incision on the back. The outer part of the cannula is closed off with a plastic seal and metal cover cap, which allows for sterility and protection of the catheter base. Flunixin $(2.5 \mathrm{mg} / \mathrm{kg}$, s.c.) is administered as an analgesic and cefazolin $(330 \mathrm{mg} / \mathrm{kg}$, i.m.) as an antibiotic. Rats are allowed to recover for a week before any SA. They are monitored and flushed daily with heparinized saline $(10 \mathrm{U} / \mathrm{ml}$ of heparin sodium; American Pharmaceutical Partners) in 0.9\% bacteriostatic sodium chloride (Hospira) that contains $52.4 \mathrm{mg} / 0.2 \mathrm{ml}$ of cefazolin. Catheter patency is tested (at least at the start and end of the experiment) with brevital sodium (1.5 mg, i.v.), a short-acting barbiturate. Only rats that pass the test (immediate loss of muscle tone) are included in GWAS and the biobanks. In each cohort, a number of rats were not surged and subsequently not exposed to drugs. These naive rats are from the same cohort (same or different breeder pairs) as the drug-exposed animals and thus age-matched control animals.

\section{Drugs}

Oxycodone (Sigma-Aldrich) is dissolved in $0.9 \%$ sterile saline (Hospira) and administered at $150 \mu \mathrm{g} / \mathrm{kg}$ per infusion intravenously. Cocaine $\mathrm{HCl}$ (National Institute on Drug Abuse, Bethesda, MD) is dissolved in $0.9 \%$ sterile saline and administered intravenously at a dose of $0.5 \mathrm{mg} / \mathrm{kg}$ per infusion.

\section{Behavioral testing}

All behavioral tests were performed in the dark cycle of the animals. Testing took place while the animals were 715 weeks old. Naive rats underwent the behavioral tests that did not require a catheter.

\section{Operant SA}

SA is performed in operant conditioning chambers $(29 \times 24 \times 19.5 \mathrm{~cm}$; Med Associates) enclosed in lit, sound-attenuating, ventilated environmental cubicles. The front door and back wall of the chambers are constructed of transparent plastic, and the other walls are opaque metal. Each chamber is equipped with two retractable levers that are located on the front panel. Each session is initiated by the extension of two retractable levers into the chamber. Drugs (cocaine, $0.5 \mathrm{mg} / \mathrm{kg}$ per infusion in saline or oxycodone, $0.15 \mathrm{mg} / \mathrm{kg}$ per infusion in saline) is delivered through plastic catheter tubing connected to an infusion pump. The infusion pump is activated by responses on the right (active) lever that is reinforced on a fixed ratio (FR)1 schedule, with the delivery of $0.1 \mathrm{ml}$ of the drug per lever press over $6 \mathrm{~s}$ followed by a 20-s time-out period that is signaled by the illumination of a cue light above the active lever and during which active lever presses do not result in additional infusions. Responses on the left (inactive) lever are recorded but have no scheduled consequences. Fluid delivery and behavioral data recording are computer-controlled. Initially, rats are familiarized and trained to self-administer during ShA sessions (ten sessions of $2 \mathrm{~h}$ for cocaine and four sessions of $2 \mathrm{~h}$ for oxycodone). The actual SA experiment gives longer access (LgA, 14 sessions of $6 \mathrm{~h}$ for cocaine and $12 \mathrm{~h}$ for oxycodone). Five 
sessions are performed per week, with a break over the weekend.

\section{Motivation: progressive ratio (PR) responding (both cohorts)}

Rats are tested on a PR schedule of reinforcement, in which the response requirements for receiving a single reinforcement increases according to the following equation: [5e(injection number $\times 0.2)$ ] -5 , which results in: 1,2 , $4,6,9,12,15,20,25,32,40,50,62,77,95,118,145$, $178, \ldots$ for cocaine and $1,1,2,2,3,3,4,4,5,5,6,6,7,7$, $8,8,9,9,10,10,11,12,13,14, \ldots$ for oxycodone. The break point is defined as the last ratio attained by the rat before a 60-min period during which a ratio is not completed, at which point the program automatically terminates. PR is performed after ShA and LgA, besides after footshock in the cocaine cohorts.

\section{Irritability-like behavior: bottle brush test (cocaine cohorts only)}

Testing consists of ten 10-s trials with 10-s intertrial intervals in plastic cages $(27 \times 48 \times 20 \mathrm{~cm})$ with clean bedding. The rat is placed in the back of the cage, and a bottlebrush was rotated rapidly toward its whiskers. Three trained observers record in real-time both aggressive responses (smelling, biting, boxing, following, and exploring the bottlebrush) and defensive responses (escaping, digging, jumping, climbing, defecation, vocalization, and grooming). Total aggressive and defensive scores are calculated for each animal based on the average score of the observers. Both aggressive and defensive behaviors are summed to calculate the total irritability score. Irritability-like behavior reflects a composite measure of aggressive versus defensive responses. The test is performed after surgery (baseline) and after LgA $18 \mathrm{~h}$ in withdrawal.

\section{Mechanical nociception: von Frey test (oxycodone cohorts only)}

Hind paw withdrawal thresholds (time and force) are determined $12 \mathrm{~h}$ into withdrawal using a dynamic plantar aesthesiometer for mechanical stimulation (electronic Von Frey, Ugo Basile), which automates Von Frey measurements. The needle of the device is placed to touch under the back paw of the rat (who sits in a box on a metal grid), and then the force increases at a constant rate (from 0 to 40 in 20 s) until the rat lifts its paw. Time and force for paw lifting are recorded. The measurement is repeated $3 \times$ for each hind paw and averaged. The test is performed before surgery (baseline) and in withdrawal $12 \mathrm{~h}$ after the last LgA session.

\section{Compulsive-like responding: contingent footshock (co- caine cohorts only)}

During this FR1 SA protocol, $30 \%$ of active lever presses are randomly associated with a footshock $(0.3 \mathrm{~mA}$ for $0.5 \mathrm{~s})$ through the cage's floor grid. This test is only performed in the cocaine cohort after LgA, as the analgesic effect op opioids could confound this measure.
Opioid-induced analgesia: tail immersion test (oxycodone cohorts only)

The rat is secured in a towel and its tail tip immersed in hot water $\left(52^{\circ} \mathrm{C}\right)$. The latency to lift the tail out of the water is recorded. This test is performed before surgery without any opioid administration (baseline), and after administering oxycodone $(0.15 \mathrm{mg} / \mathrm{kg}$, i.v. $)$ both before the start of the first ShA session and before the last LgA session to evaluate the development of tolerance to the analgesic effect of oxycodone during SA.

\section{Medication testing (oxycodone cohorts only)}

Rats are treated with intraperitoneal injections of buprenorphine $(0.5 \mathrm{mg} / \mathrm{kg})$, methadone $(3 \mathrm{mg} / \mathrm{kg})$, naloxone $(3 \mathrm{mg} / \mathrm{kg}$ ), and vehicle $30 \mathrm{~min}$ before a PR session using a within-subject Latin square design. The drug test days are separated by a LgA session without treatment.

\section{Biological sampling}

All samples are stored at $-80^{\circ} \mathrm{C}$.

\section{Longitudinal samples}

Fecal pellet collection. Feces (two droppings on average) are collected fresh from the animal in a sterile tube by gently massaging the rectal canal and snap-frozen on dry ice. Fecal pellet collection is performed before surgery (baseline), after short access (ShA), and after long access (LgA). For cocaine: post-ShA feces are collected right after the session finished (intoxication), while post-LgA feces are collected 18-24 $\mathrm{h}$ after finishing the session (withdrawal). A limited set of feces was also collected in abstinence. For oxycodone: all feces are collected in withdrawal, $16-18 \mathrm{~h}$ for ShA and 7-8 $\mathrm{h}$ for LgA.

Blood collection. While the rats are anesthetized for the intravenous catheter surgery, 200- to 400- $\mu$ l blood (baseline) is collected through retroorbital bleed in EDTAcoated tubes that are immediately inverted five times. Blood samples are processed within $1 \mathrm{~h}$ of collection by centrifugation at $2000 \times g$ at room temperature (RT) for 10 $\mathrm{min}$ to pellet the erythrocytes. The supernatant plasma is immediately transferred into a fresh tube, scored for quality on a scale from 0 to 5 , snap-frozen on dry ice, and stored at $-80^{\circ} \mathrm{C}$.

Urine collection. Urine $(>100 \mu \mathrm{l})$ is collected from rats isolated overnight on a grid over non-absorbent sand at baseline and in acute withdrawal after LgA.

Vaginal smear. Cells from the vaginal opening of female rats are collected with a wet cotton $Q$ swab and dabbed onto a glass microscope slide to determine where the females are in their estrous cycle (proestrus, estrus, metestrus, or diestrus) at baseline, after the last ShA and LgA, and before dissection.

\section{Terminal samples}

After the last $\operatorname{LgA}$ session and completing the behavioral characterization, rats are killed at three different timepoints. (1) Intoxication: euthanasia is performed during the last SA session (after $\sim 2 \mathrm{~h}$ of drug access). (2) Acute withdrawal: euthanasia is performed $18 \mathrm{~h}$ (for cocaine) and $12 \mathrm{~h}$ (for oxycodone) after the end of the last SA session. (3) Protracted abstinence: euthanasia is 

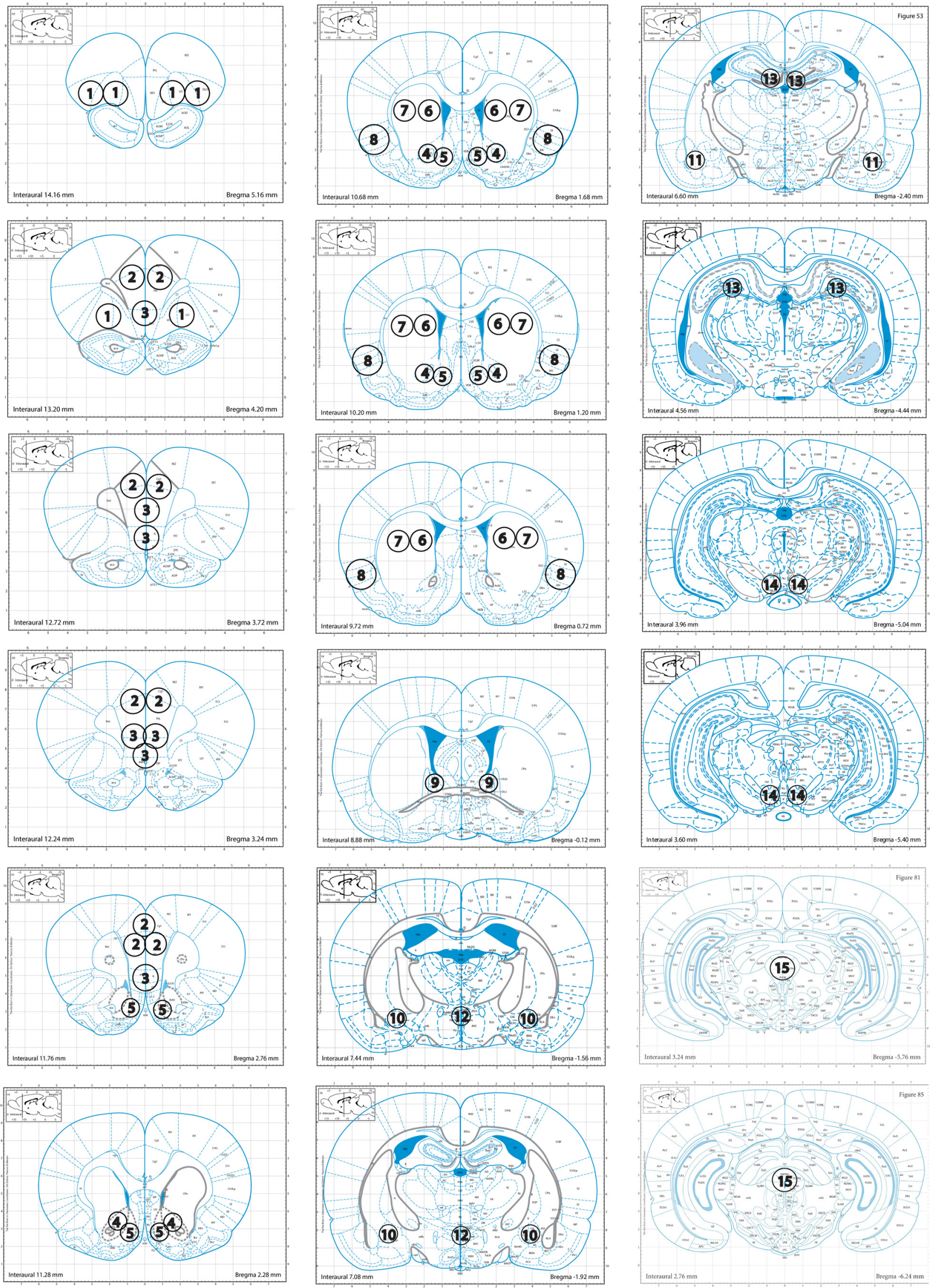
performed four to five weeks after the last SA session. The age of animals at killing is $\sim 16-18$ weeks for the intoxication and acute withdrawal timepoints and 2123 weeks for the protracted abstinence time point. For quality control, audio recordings are made during the dissections. Rats are anesthetized with $\mathrm{CO}_{2}$, followed by the following.

(1) Blood collection $(\sim 8 \mathrm{ml})$ via cardiac puncture in EDTA coated tubes that are immediately inverted five times. For plasma, up to $3 \mathrm{ml}$ of blood is transferred to sterile Eppendorf tubes, processed as above, and snapfrozen in two aliquots of $\sim 0.5 \mathrm{ml}$. PBMCs are also prepared from the blood in a tissue culture hood. First, whole blood $(2 \mathrm{ml})$ is diluted with an equal volume $(2 \mathrm{ml})$ of freshly prepared Tris-buffered saline (TBS; $10 \mathrm{~mm}, \mathrm{pH} 7$ ) with $2 \%$ fetal bovine serum (FBS). This diluted blood $(4 \mathrm{ml})$ is then carefully layered over $2 \mathrm{ml}$ of density barrier (TBS diluted in $\mathrm{H}_{2} \mathrm{O}$ at 2.5:0.5 mixed with OptiPrep in a 2.7:9.3 ratio, to achieve a final osmolarity of $242 \pm 10$ mOsm) in a $12 \mathrm{ml}$ Falcon tube. Tubes are centrifuged at $700 \times g$, RT for 30 min with slow acceleration and deceleration to prevent disruption of the interface. After separation, PBMCs can be collected and diluted in an equal amount $(\sim 200 \mu \mathrm{l})$ of TBS with $2 \%$ FBS. Cells are pelleted by centrifugation at $400 \times g$, RT for $10 \mathrm{~min}$. The pellet is scored for quantity on a scale from 0 to 3 , resuspended in $250 \mu \mathrm{l}$ Cytostor10 (Stemcell Technologies), transferred into a cryotube, and slowly frozen to $-80^{\circ} \mathrm{C}$.

(2) Brains are extracted from the skull and submerged in a slurry of 2-methylbutane with dry ice $\left(\right.$ at $\left.-30^{\circ} \mathrm{C}\right)$ for about $8 \mathrm{~s}$, till fully frozen. Brains are put in a container on dry ice till final storage at $-80^{\circ} \mathrm{C}$. Punches from selected regions are collected from $500-\mu \mathrm{m}$ brain slices according to the maps in Figure 1. They are kept frozen at all times and stored at $-80^{\circ} \mathrm{C}$.

(3) Other organs. Heart, liver (left and right median lobe), kidney (left and right), adrenal gland (left and right), ovary (left and right), or testicles (left and right) are dissected out, rinsed in PBS, weighed, and snap-frozen in their respective containers on dry ice. Colon, cecum + poop, and tail tip are dissected and snap-frozen in their respective containers on dry ice. The dissected spleen is combined with the RFID chip in PBS in its tube that is snapfrozen on dry ice. The spleen is used for genotyping. The tail tip (3/4") is stored as back-up material for that purpose.

Alternatively, rats are killed under anesthesia by exsanguination (blood was collected from the trunk and further processed as described above) and perfusion with $100 \mathrm{ml}$ of PBS followed by $400 \mathrm{ml}$ of $4 \%$ paraformaldehyde. The brains and other organs are then postfixed in paraformaldehyde overnight and transferred to $30 \%$ sucrose in PBS/ $0.1 \%$ azide solution at $4^{\circ} \mathrm{C}$ for $2-3 \mathrm{~d}$, before freezing and storing at $-80^{\circ} \mathrm{C}$.

\section{Data analysis}

Z-scores are used to reduce sex and cohort effects and scale the different behavioral paradigm outputs. They are calculated as follows $\mathrm{Z}=(\mathrm{x}-\mu) / \sigma$, where $\mathrm{x}$ is the raw value, $\mu$ is the mean of the cohort, and $\sigma$ is the standard deviation of the cohort. Z-scores are calculated for the following measures:

Escalation index: $E=Z_{f} / Z_{0}$, where $Z_{f}$ is the $Z$-score of an animal on the last $3 \mathrm{~d}$ of escalation and $Z_{0}$ is the $Z$ score of an animal during the first day of escalation. A daily Escalation Index $\left(E_{i}\right)$ can be calculated for each session $\left(E_{i}=Z_{i} / Z_{0}\right)$, where $Z_{i}$ is the $Z$-score on a given escalation session $i$. This index can also be calculated for the first hour or the entire session. Pain index (withdrawal): Zscores are calculated from the pain threshold in withdrawal as a percentage of the baseline threshold. Irritability index (withdrawal): Z-score of the total irritability score with the baseline scores subtracted. PR index (motivation): Z-score of the breakpoint after LgA. Shock index (compulsivity): Z-score of the number of infusions. Tolerance index (intoxication): Z-scores are calculated from the difference in latency to lift the tail from a hot water bath after a dose of oxycodone between the end of $\mathrm{LgA}$ and before the first ShA session.

The addiction index (Al) is obtained by averaging relevant behavioral indexes.

\section{Results}

\section{Characterization of addiction-like behaviors}

The timeline of our standard protocols for both cocaine and oxycodone addiction-like behavior characterization is shown in Figure 2. Following surgery and one week of recovery, rats are given ShA to the drug for training $(10 \times$ 2-h sessions for cocaine at $500 \mu \mathrm{g} / \mathrm{kg}$ per infusion and $4 \times 2$-h sessions for oxycodone at $150 \mu \mathrm{g} / \mathrm{kg}$ per infusion). Then $14 \mathrm{~d}$ of LgA drug SA followed (6-h sessions for cocaine and 12-h sessions for oxycodone; same doses). Longitudinal biological samples including blood, urine, and feces are collected at three timepoints during behavioral testing: (1) at BSL before drug exposure; (2) after ShA (only feces) and; (3) after LgA (Table 1).

During ShA and LgA, which follow a FR1 schedule of reinforcement, drug intake is measured. An essential step in the characterization of addiction-like behaviors is determining the escalation of drug intake. While some rats escalate, others show no escalation or even decrease intake. Importantly, HS rats exhibit high interindividual differences and low intraindividual variability. Also important are motivation and compulsive-responding for drug, evaluated respectively using an exponential increase in cost with a PR schedule (after ShA and LgA) and an adverse

continued

Figure 1. Punch maps for $1=$ orbitofrontal cortex (OFC); $2=$ dorsal and $3=$ ventral prefrontal cortex (dPFC, vPFC); $4=\mathrm{core}$ and $5=$ shell of the nucleus accumbens (NAc); $6=$ dorsal medial and $7=$ dorsal lateral striatum (DMS, DLS); $8=$ insular cortex (insula); $9=$ dorsal bed nucleus of the stria terminalis (dBNST); $10=$ central nucleus of the amygdala (CeA); $11=$ basolateral amygdala (BLA); $12=$ paraventricular nucleus $(\mathrm{PVN}) ; 13=$ dentate gyrus of the dorsal hippocampus (DG); $14=$ ventral tegmental area (VTA); 15 = periaqueductal gray (PAG), modified from The Rat Brain in Stereotaxic Coordinates, Ed 5, by (Paxinos and Watson, 2004). 


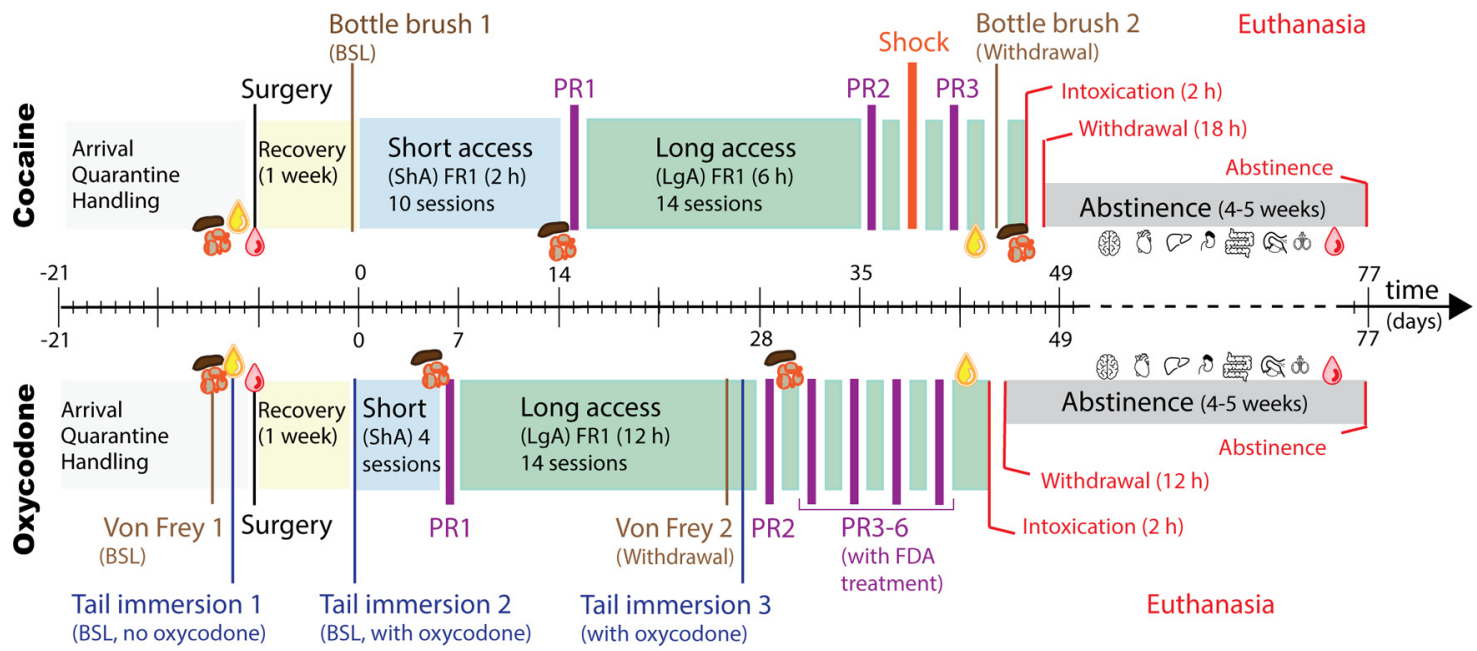

Longitudinal samples: (3) Blood Fecal pellets Urine 80 Vaginal smear

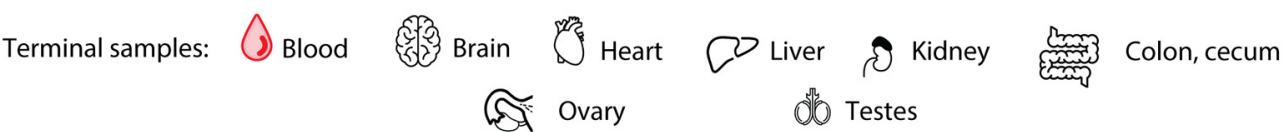

Figure 2. Experimental design of addiction-like behavior phenotyping and sampling. Timeline of behavioral tests and biological sampling relative to drug SA. Testing and sampling were performed on 6- to 15-week-old rats at baseline (BSL; before drug exposure and surgery), after ShA, and LgA to the drug, as well as at euthanasia either during intoxication, acute withdrawal (16-18 weeks old) or after prolonged abstinence (21-23 weeks old). The timeline starts with three- to four-week-old rats (shipped from WFU and quarantined at TSRI or UCSD for two weeks). The longitudinal samples collected include blood, feces, urine, and vaginal smears. The terminal samples include brain, heart, liver, kidney, colon, cecum, adrenal glands, ovaries, and testicles. The behavioral measures include hyperalgesia using the von Frey test (for oxycodone) at LgA compared with BSL, irritability-like behavior using the bottlebrush test (for cocaine) at LgA compared with BSL, opioid-induced analgesia, and tolerance using the tail immersion test (for oxycodone) before ShA and after LgA with oxycodone on board relatively compared with BSL and BSL with oxycodone, motivation with PR test (for both oxycodone and cocaine) after ShA and LgA (and after shock for cocaine only), compulsivity with FR1 using contingent footshocks (for cocaine) and the effect of FDA-approved treatments after LgA on PR tests (for oxycodone).

consequence (contingent footshock after LgA; for cocaine; Deroche-Gamonet et al., 2004; Chen et al., 2013). Withdrawal severity is evaluated by irritability-like behavior (bottlebrush test after LgA compared with BSL; for cocaine; Kimbrough et al., 2017) and hyperalgesia/allodynia (von Frey test after LgA compared with BSL; for oxycodone; Chaplan et al., 1994). For oxycodone, analgesia and tolerance are evaluated (tail immersion test) in addition to the effects of FDA-approved treatments (buprenorphine, methadone, and naltrexone with PR responding).

Animals can be characterized based on individual behavioral measures or a composite $\mathrm{Al}$ as adapted from Deroche-Gamonet et al. (2004) and (Belin et al. (2009), that classifies them as vulnerable (high addiction-like behavior; HA) with high Al versus resilient (low addiction-like behavior; LA) with low Al by a median-split. For cocaine the $\mathrm{Al}$ is the average of the Z-scores of escalation (intake during LgA FR1), motivation (intake during PR) and compulsivity (intake despite footshock). For oxycodone, the Z-scores of escalation, motivation, and hyperalgesia (reduced pain threshold during withdrawal) are averaged. Figure 3 shows the raw data for these behavioral measures for HA and LA obtained from one cohort each.

\section{Currently available samples in the biobanks}

Terminal samples (e.g., brains and other organs) are available from different rats at three timepoints (Fig. 2; Table 1): (1) after $2 \mathrm{~h}$ of SA, to study the effect of drug intoxication; (2) after $12 \mathrm{~h}$ (for oxycodone) or $18 \mathrm{~h}$ (for cocaine) past the end of the last SA session to study the effect of withdrawal; (3) after four to five weeks of protracted abstinence in the home cage to evaluate longlasting biological changes (Garavan et al., 2013). The animals are assigned to each time point in a pseudorandomized fashion to ensure a representative distribution of HA and LA rats. Additionally, we maintain a group of age-matched control animals that are not exposed to drug.

For maximal compatibility with the widest variety of potential experiments, samples are preserved with or without fixation. Traditional histology and anatomy studies do better on perfused tissues that are cleared of blood and crosslinked with paraformaldehyde (Gage et al., 2012) because this preserves the anatomic organization of the tissue and has a very long shelf-life (>10 years). However, those crosslinks disturb many molecular biology protocols, which work better with fresh, rapidly frozen tissue (snap-frozen; Auer et al., 2014). Alternatively, structurally 
Table 1: Summary of the composition of the biobank samples, giving the number of animals per sample, grouped by drug, time point, and processing per sample type

\begin{tabular}{|c|c|c|c|c|c|c|c|c|}
\hline \multicolumn{2}{|c|}{ Number of animals } & \multicolumn{3}{|l|}{ Cocaine } & \multicolumn{3}{|l|}{ Oxycodone } & \multirow[t]{2}{*}{ Number of samples per animal } \\
\hline Longitudinal & Sample & BSL & ShA & $\operatorname{LgA}$ & BSL & ShA & $\operatorname{LgA}$ & \\
\hline & Urine & 418 & - & 360 & 421 & - & 351 & 1 per timepoint \\
\hline & Feces & 714 & 418 & 628 & 421 & 273 & 285 & 1 per timepoint \\
\hline & Plasma & 418 & - & Terminal & 421 & - & Terminal & 1 per timepoint \\
\hline \multirow[t]{12}{*}{ Terminal } & Sample & Intoxication & Withdrawal & Abstinence & Intoxication & Withdrawal & Abstinence & \\
\hline & Brain & 167/36 (snap/PFA) & 160/36 (snap/PFA) & 199/21 (snap/PFA) & 82 (snap) & 85 (snap) & 184 (snap) & $1-15$ (whole brain or $\sim 15$ regions) \\
\hline & Heart & 150/36 (snap/PFA) & 144/36 (snap/PFA) & 125/21 (snap/PFA) & 82 (snap) & 85 (snap) & 78 (snap) & 1 \\
\hline & Liver & 150/36 (snap/PFA) & 144/36 (snap/PFA) & 125/21 (snap/PFA) & 82 (snap) & 85 (snap) & 78 (snap) & 2 (right, left) \\
\hline & Kidney & 150/36 (snap/PFA) & 144/36 (snap/PFA) & 125/21 (snap/PFA) & 82 (snap) & 85 (snap) & 78 (snap) & 2 (right, left) \\
\hline & Adrenal Gland & 150/36 (snap/PFA) & 144/36 (snap/PFA) & 125/21 (snap/PFA) & 82 (snap) & 85 (snap) & 78 (snap) & 2 (right, left) \\
\hline & Ovary & 72/18 (snap/PFA) & 66/17 (snap/PFA) & 60/12 (snap/PFA) & 43 (snap) & 40 (snap) & 38 (snap) & 2 (right, left) \\
\hline & Testes & 78/18 (snap/PFA) & 78/19 (snap/PFA) & 65/9 (snap/PFA) & 39 (snap) & 45 (snap) & 40 (snap) & 2 (right, left) \\
\hline & Colon & 150/36 (snap/PFA) & 144/36 (snap/PFA) & 125/21 (snap/PFA) & 82 (snap) & 85 (snap) & 78 (snap) & 1 \\
\hline & Cecum & 150/36 (snap/PFA) & 144/36 (snap/PFA) & 125/21 (snap/PFA) & 82 (snap) & 85 (snap) & 78 (snap) & 1 \\
\hline & Plasma & 203 (snap) & 196 (snap) & 147 (snap) & 82 (snap) & 85 (snap) & 106 (snap) & 2 aliquots \\
\hline & PBMCs & 158 (snap) & 151 (snap) & 101 (snap) & - & - & - & 1 \\
\hline
\end{tabular}

Samples from naive animals are included in the given number and represent $\sim 20 \%$ of samples. Snap, snap-frozen; PFA, paraformaldehyde.

intact living cells are cryopreserved to keep cells viable with a long shelf-life. PBMCs are extracted from whole blood and slowly frozen with cryoprotectant (Rasooly et al., 2017). PBMCs can be used to prepare induced pluripotent stem cells (iPSCs) and thus virtually any other cell type (Takahashi and Yamanaka, 2006; Hokayem et al., 2016).

The two biobanks currently have over 20,000 samples from $\sim 1,100$ unique animals, aiming to grow to double in size. Brains can be requested as a whole, sliced, or punched from at least 15 different brain regions (Fig. 1). All samples are stored at $-80^{\circ} \mathrm{C}$ in multiple freezers, located in different buildings with back-up generators to limit loss in case of adverse events. Freezer temperatures are monitored online with an alarm and notification service (Minus80 monitoring; https://minus80monitoring. com/).

\section{The biobank ecosystem}

The biobanks are currently collaborating on 27 independent research projects with major universities and research institutes in the United States and Europe, to which we have shipped out $>1000$ samples (Table 2). Current and past collaborative projects have used brain tissue for circuitry analysis, histology, proteomics, epigenetic analysis, and single-cell sequencing. Plasma is being used for exosome transcriptomics, metabolomics, and biomarker discovery. Feces have been analyzed in microbiome projects and for biomarker discovery. Pelvic floor muscle and semen were dissected by special request to study oxycodone's and cocaine's effect on their composition, respectively. Publications that result from the use of biobank samples will be tracked on the websites (https://www.cocainebiobank.org/ and https://www. oxycodonebiobank.org/).

An application example was recently published (Kallupi et al., 2020). Kallupi and colleagues were interested in investigating nociceptin/orphanin FQ peptide (N/OFQ, nociceptin) levels in the CeA of rats with high versus low addiction profiles for oxycodone to confirm a preliminary hypothesis. N/OFQ is an endogenous opioid-like peptide with an important role in opioid tolerance and reward (Ciccocioppo et al., 2000). Analysis of CeA punches from the oxycodone biobank selected with a high $(\mathrm{HA}, N=7)$ or low (LA, $N=7$ ) addiction phenotype based on their $\mathrm{Al}$ and naive controls $(N=7)$ by Western blot analysis (Fig. 4A) showed a significant reduction of the peptide in $H A$ rats compared with naive rats (one-way ANOVA: $p=0.02$, post hoc: $p=0.02$; Fig. $4 B$ ). Moreover, individual N/OFQ levels correlated with the animals' $\mathrm{Al}(r=-0.62, p=0.017$; Fig. $4 C)$. These results confirmed that the N/OFQ levels in the $\mathrm{CeA}$ inversely correlate with addiction-like behaviors and illustrate these genetically-diverse samples' utility.

\section{Discussion}

This report describes the cocaine and oxycodone biobanks, two biological repositories aimed at facilitating research on the biological mechanisms underlying addiction. Every sample in the biobanks can be selected based on phenotype or genotype and comes with a comprehensive report of our behavioral and genetic characterization. The report also includes the animal identification number, sex, age, body weight, the weight of the sample, collection date, and time from anoxia to freezing. Samples are available both longitudinally and cross-sectionally, tracked using RFID chips and barcodes to ensure tracing and transparency. Longitudinal samples currently include blood plasma, feces, and urine collected at baseline, after ShA, and LgA (Fig. 2; Table 1). Organs and other terminal samples are harvested during drug intoxication, acute withdrawal, or protracted abstinence or from naive controls (Fig. 2; Table 1). Ongoing collaborations and other potential applications include omics-studies and neuroanatomy, biomarker discovery, and other cellular and molecular approaches (Table 2). To maximize compatibility with this variety of downstream applications, samples are cryopreserved, fixed, or snapfrozen. 
A

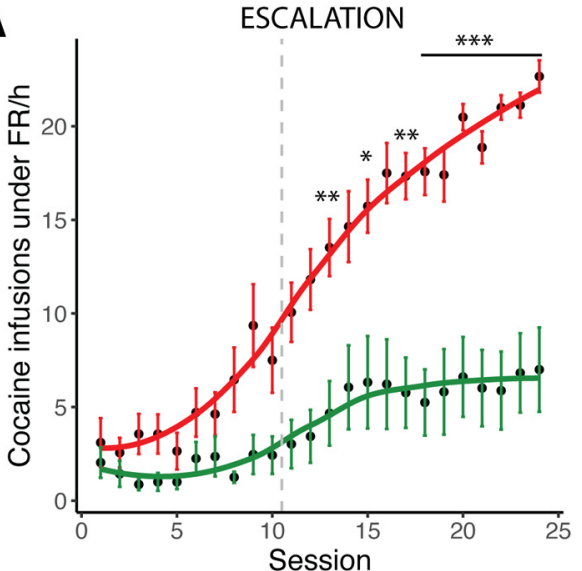

D

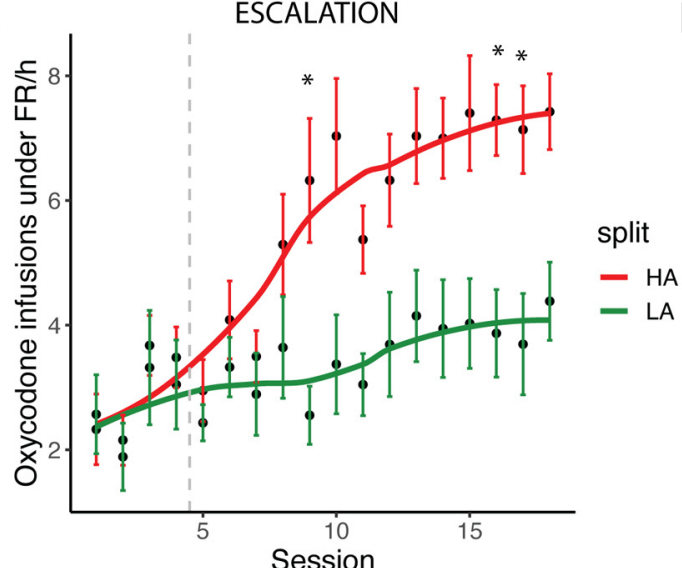

B

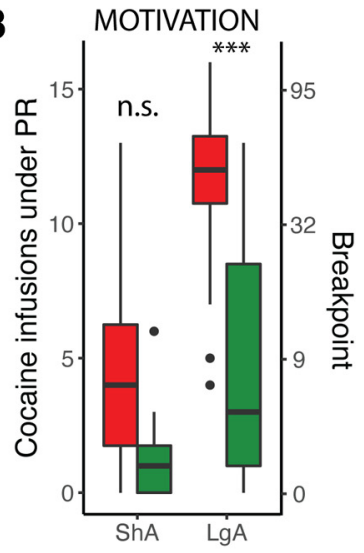

E

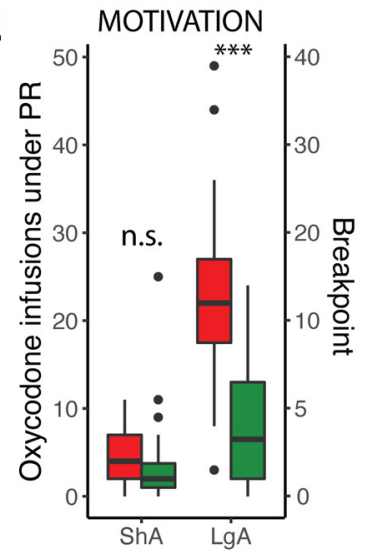

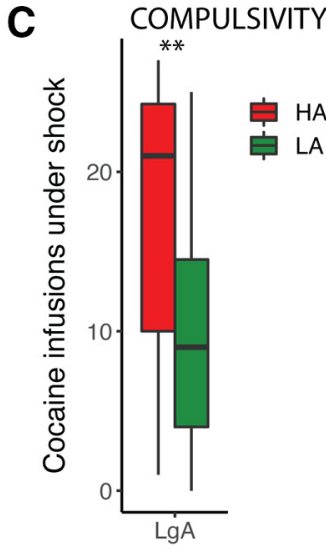

$\mathbf{F}$

F HYPERALGESIA

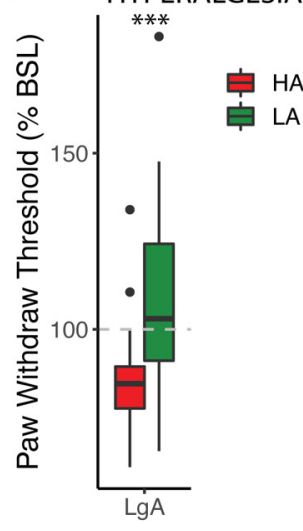

Figure 3. Characterization of addiction-like behavior with the Al. Example of addiction-like behaviors assessed in rats and used for the calculation of the Al: escalation $(\boldsymbol{A})$, motivation $(\boldsymbol{B})$, and compulsivity $(\boldsymbol{C})$ for cocaine $(N=39$, cohort 04$)$ or escalation $(\boldsymbol{D})$, motivation $(\boldsymbol{E})$, and hyperalgesia $(\boldsymbol{F})$ for oxycodone $(N=52$, cohort 06$)$. The data are represented following a median split of the cohort's Al with animals with high addiction-like behaviors $(\mathrm{HA})$ in red and low addiction-like behaviors (LA) in green. Error bars represent the standard error of the mean $(\boldsymbol{A}, \boldsymbol{D})$. Boxplots represent the median with the $75 \%$ quartile (upper hinge), $25 \%$ quartile (lower hinge), and observations smaller than or equal to upper hinge $+1.5^{*}$ interquartile range (IQR; upper whisker) or greater than or equal to lower hinge -1.5 * IQR (lower whisker) (B, C, $\boldsymbol{E}, \boldsymbol{F}$ ). Data beyond the end of the whiskers ("outlying" points) are plotted individually. Statistics represent the difference between HA and LA $\left[{ }^{*} p<0.05,{ }^{* *} p<0.02,{ }^{* *} p<0.001\right.$, not significant (n.s.) and were obtained with two-way ANOVA for each behavioral measure, using Bonferroni's multiple comparison test where appropriate].

Addiction-like behavior is characterized using multiple behavioral paradigms with standardized operating procedures (SOPs). The animal model of LgA to drug SA has shown high face, predictive/postdictive, and construct validity and is thus highly relevant to psychostimulant and opioid use disorders (George et al., 2008, 2014; Vendruscolo et al., 2011; Edwards and Koob, 2013; Wade et al., 2015). It is associated with neuroadaptations that are also observed in humans with cocaine use disorder (Briand et al., 2008; George et al., 2008). The model has been shown to exhibit at least seven of the 11 criteria for SUD listed in the DSM5 , including most of the criteria required to diagnose severe use disorder: (1) tolerance (Ben-shahar et al., 2006), (2) withdrawal (Ahmed et al., 2002; Vendruscolo et al., 2011), (3) substance taken in larger amount than intended (Ahmed and Koob, 1998), (4) unsuccessful efforts to quit (Ahmed and Cador, 2006), (5) considerable time spent to obtain the drug (Wee et al., 2008), (6) important social, work, or recreational activities given up because of use (George et al., 2008; Lenoir et al., 2013), and (7) continued use despite adverse consequences (Vanderschuren and Everitt, 2004; Xue et al., 2012). Sex differences in cocaine and oxycodone intake have been identified using this model, as seen in human, with females appearing more vulnerable (Roth and Carroll, 2004; Ramôa et al., 2013; Kimbrough et al., 2020). Behavioral testing for the Biobanks confirmed these findings with significant differences in escalation, intake, motivation and compulsivity. Samples can be selected based on sex and estrous cycle to facilitate research into these differences. Moreover, while the LgA model was only used by a few laboratories a decade ago, it has now been widely adopted in the field and is routinely used in prominent laboratories in North America and Europe. A major benefit of the biobanks, and SOPs, is the potential to reduce oftenobserved nonspecific behavioral variability between 
Table 2: Examples of ongoing collaborations with samples from the biobanks

\begin{tabular}{lll}
\hline Sample & Preservation & Application \\
\hline Brain & Snap-frozen & Proteomics \\
Brain & Perfused & Circuitry analysis \\
Brain & Perfused & Histology and cellular activation \\
Brain (+ punches) & Snap-frozen & Epigenetics and epigenomics \\
Brain (+ punches) & Snap-frozen & Single-cell sequencing \\
Semen & Snap-frozen & Semen composition \\
Muscles & Snap-frozen + OCT embedded & Muscle composition \\
Feces & Snap-frozen & Microbiome analysis \\
Feces & Snap-frozen & Biomarker discovery \\
Plasma & Snap-frozen & Metabolomics \\
Plasma & Snap-frozen & Biomarker discovery \\
Plasma & Snap-frozen & Exosome transcriptomics \\
\hline
\end{tabular}

studies because of differences in operant training, learning, drug priming, stress, food restriction, and circadian cycle.

Family and twin studies demonstrate that $\sim 50 \%$ of the vulnerability to cocaine and opioid use disorders is determined by genetic factors (Kreek et al., 2005; Agrawal et al., 2012; Ducci and Goldman, 2012). Recently, large human GWAS have begun to identify some of the genes involved in SUDs (Walters et al., 2018; Kranzler et al., 2019; Liu et al., 2019; Sanchez-Roige et al., 2019a,b, 2020; Erzurumluoglu et al., 2020; Polimanti et al., 2020; Zhou et al., 2020a,b); however, the vast majority of genes that confer risk remain unknown. Therefore, the genes/alleles that mediate individual differences in the effect of cocaine and opioids and the biological mechanisms responsible for developing addiction-like behaviors are still poorly defined (Klepstad et al., 2011; Hart et al., 2013). Taking advantage of the fact that the eight founders of the HS rats have been sequenced, HS rats can be used to map genetic loci to one or a few Mb. Whole-genome sequencing identifies hundreds of thousands of single nucleotide polymorphisms (SNPs) per individual and, following imputation, provides genotypes at millions of SNPs. Combining hundreds of animals' genetic and genomic data will feed a subsequent GWAS to identify gene variants contributing to vulnerability and resilience to SUD-like behavior (Chitre et al., 2020), which forms the basis to identify specific genetic loci, estimate heritability, investigate genetic correlations and perform phenome- wide association studies (PheWAS) and transcriptomewide association studies (TWAS). All of which may identify novel gene variants to inform human personalized diagnosis and treatment. Further expansion of the biobank ecosystem also aims to facilitate the integration of genetic complexity studies into other fields of addiction neuroscience.

Certain difficulties associated with clinical studies, including imprecision of self-reported drug addiction measures, the lack of longitudinal analyses of behavior, lack of biological samples, the lack of control of environmental variables, and qualitative versus quantitative measures, can be overcome through the use of animal models. Preclinical settings allow for longitudinal analyses with qualitative and quantitative measures that can be highly standardized and replicated while minimizing the variability because of the environment. Such a strategy has the potential to reveal a greater number of gene variants, metabolites, protein regulations, and neuroadaptations associated with the vulnerability to develop compulsive cocaine or oxycodone use. Moreover, because we have so many animals, these repositories will facilitate replication, validation, and follow-up studies that are virtually impossible in human subjects and extremely difficult in regular small-size [at least one or two order(s) of magnitude lower than current] preclinical studies. Despite the large number of animals in the biobank, grouping the samples in a biobank helps reduce the total number of lab animals and is thus beneficial for animal welfare. Such a
A

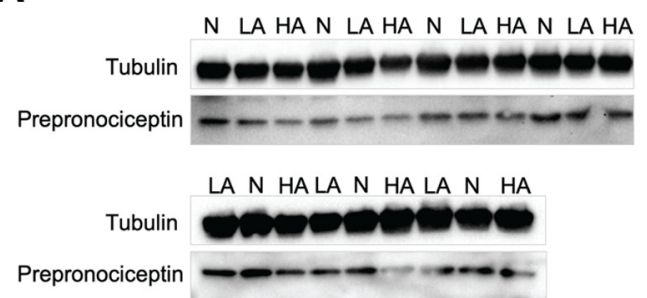

B

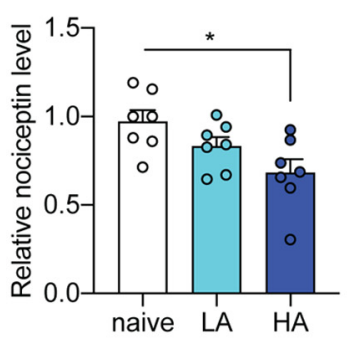

C

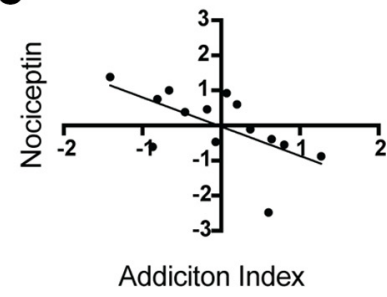

Figure 4. Application example looking at N/OFQ levels in oxycodone biobank brain punches from rats with different addiction profiles. $\boldsymbol{A}$, Immunoluminescent Western blottings that show nociceptin levels in naive, LA, and HA rats; $n=21$ ( $n=7$ per group). $\boldsymbol{B}$, Western blot analysis revealed a significant decrease in nociceptin levels (internally normalized to $\beta$-tubulin and a naive rat on the blot) in HA rats compared with naive rats; ${ }^{\star} p=0.02$ one-way ANOVA, error bars represent standard error of mean. $\boldsymbol{C}$, Correlation between Al and the Z-score of nociceptin levels in the $\mathrm{CeA} ;{ }^{*} p<0.02$ pearson correlation. Partial figure reproduced from Kallupi et al. (2020). 
resource is also cost-effective to the scientific community and will facilitate addiction research by making samples available to researchers who do not have the capability to perform advanced behavioral and genetic analysis.

\section{References}

Agrawal A, Verweij KJH, Gillespie NA, Heath AC, Lessov-Schlaggar CN, Martin NG, Nelson EC, Slutske WS, Whitfield JB, Lynskey MT (2012) The genetics of addiction-a translational perspective. Transl Psychiatry 2:e140.

Ahmed S, Koob G (1998) Transition from moderate to excessive drug intake : change in hedonic set point. Science 282:298-300.

Ahmed SH, Cador M (2006) Dissociation of psychomotor sensitization from compulsive cocaine consumption. Neuropsychopharmacology 31:563-571.

Ahmed SH, Kenny PJ, Koob GF, Markou A (2002) Neurobiological evidence for hedonic allostasis associated with escalating cocaine use. Nat Neurosci 5:625-626.

Auer H, Mobley JA, Ayers LW, Bowen J, Chuaqui RF, Johnson LA, Livolsi VA, Lubensky IA, McGarvey D, Monovich LC, Moskaluk CA, Rumpel CA, Sexton KC, Washington MK, Wiles KR, Grizzle WE, Ramirez NC (2014) The effects of frozen tissue storage conditions on the integrity of RNA and protein. Biotech Histochem 89:518528.

Baud A (2013) Combined sequence-based and genetic mapping analysis of complex traits in outbred rats. Nat Genet 45:767-775.

Belin D, Balado E, Piazza PV, Deroche-Gamonet V (2009) Pattern of intake and drug craving predict the development of cocaine addiction-like behavior in rats. Biol Psychiatry 65:863-868.

Ben-shahar O, Moscarello JM, Ettenberg A (2006) One hour, but not six hours, of daily access to self-administered cocaine results in elevated levels of the dopamine transporter. Brain Res 1095:148153.

Briand LA, Flagel SB, Seeman P, Robinson TE (2008) Cocaine selfadministration produces a persistent increase in dopamine D2 high receptors. Eur Neuropsychopharmacol 18:551-556.

Chaplan SR, Bach FW, Pogrel JW, Chung JM, Yaksh TL (1994) Quantitative assessment of tactile allodynia in the rat paw. J Neurosci Methods 53:55-63.

Chen BT, Yau HJ, Hatch C, Kusumoto-Yoshida I, Cho SL, Hopf FW, Bonci A (2013) Rescuing cocaine-induced prefrontal cortex hypoactivity prevents compulsive cocaine seeking. Nature 496:359362.

Chitre AS, Polesskaya O, Holl K, Gao J, Cheng R, Bimschleger H, Garcia Martinez A, George T, Gileta AF, Han W, Horvath A, Hughson A, Ishiwari K, King CP, Lamparelli A, Versaggi CL, Martin C, St Pierre CL, Tripi JA, Wang T, et al. (2020) Genome-wide association study in 3,173 outbred rats identifies multiple loci for body weight, adiposity, and fasting glucose. Obesity 28:1964-1973.

Ciccocioppo R, Angeletti S, Paolo P, Weiss F, Massi M (2000) Effect of nociceptin $r$ orphanin $F Q$ on the rewarding properties of morphine. Eur J Pharmacol 404:153-159.

de Cid R, Fonseca F, Gratacòs M, Gutierrez F, Martín-Santos R, Estivill X, Torrens M (2008) BDNF variability in opioid addicts and response to methadone treatment: preliminary findings. Genes Brain Behav 7:515-522.

Deroche-Gamonet V, Belin D, Piazza PV (2004) Evidence for addiction-like behavior in the rat. Science 305:1014-1017.

Ducci F, Goldman D (2012) The genetic basis of addictive disorders. Psychiatr Clin North Am 35:495-519.

Eap CB, Buclin T, Baumann P (2002) Interindividual variability of the clinical pharmacokinetics of methadone: implications for the treatment of opioid dependence. Clin Pharmacokinet 41:1153-1193.

Edwards S, Koob GF (2013) Escalation of drug self-administration as a hallmark of persistent addiction liability. Behav Pharmacol 24:356-362.

Erzurumluoglu AM, Liu M, Jackson VE, Barnes DR, Datta G, Melbourne CA, Young R, Batini C, Surendran P, Jiang T, Adnan
SD, Afaq S, Agrawal A, Altmaier E, Antoniou AC, Asselbergs FW, Baumbach C, Bierut L, Bertelsen S, Boehnke M, et al. (2020) Meta-analysis of up to 622,409 individuals identifies 40 novel smoking behaviour associated genetic loci. Mol Psychiatry 25:2392-2409.

Gage GJ, Kipke DR, Shain W (2012) Whole animal perfusion fixation for rodents. J Vis Exp. Advance online publication. Retrieved July 30, 2012. doi: 10.3791/3564.

Garavan H, Brennan K, Hester R, Whelan R (2013) The neurobiology of successful abstinence. Curr Opin Neurobiol 23:668-674.

George O, Mandyam CD, Wee S, Koob GF (2008) Extended access to cocaine self-administration produces long-lasting prefrontal cortex-dependent working memory impairments. Neuropsychopharmacology 33:2474-2482.

George O, Koob GF, Vendruscolo LF (2014) Negative reinforcement via motivational withdrawal is the driving force behind the transition to addiction. Psychopharmacology (Berl) 231:3911-3917.

Grant BF, Saha TD, Ruan WJ, Goldstein RB, Chou SP, Jung J, Zhang H, Smith SM, Pickering RP, Huang B, Hasin DS (2016) Epidemiology of DSM-5 drug use disorder: results from the national epidemiologic survey on alcohol and related conditions-III. JAMA Psychiatry 73:39-47.

Hansen C, Spuhler K (1984) Development of the National Institutes of Health genetically heterogeneous rat stock. Alcohol Clin Exp Res 8:477-479.

Hart AB, De Wit H, Palmer AA (2013) Candidate gene studies of a promising intermediate phenotype: failure to replicate. Neuropsychopharmacology 38:802-816.

Hokayem JE, Cukier HN, Dykxhoorn DM (2016) Blood derived induced pluripotent stem cells (iPSCs): benefits, challenges and the road ahead. J Alzheimers Dis Parkinsonism 6:275.

Kallupi M, Carrette LLG, Kononoff J, Woods LCS, Palmer AA, Schweitzer P, George O, De Guglielmo G (2020) Nociceptin attenuates the escalation of oxycodone self-administration by normalizing CeA-GABA transmission in highly addicted rats. Proc Natl Acad Sci USA 117:2140-2148.

Keele GR (2018) Variants for adiposity traits in outbred rats. Obesity 26:213-222.

Kimbrough A, de Guglielmo G, Kononoff J, Kallupi M, Zorrilla EP, George O (2017) CRF(1) receptor-dependent increases in irritability-like behavior during abstinence from chronic intermittent ethanol vapor exposure. Alcohol Clin Exp Res 41:1886-1895.

Kimbrough A, Kononoff J, Simpson S, Kallupi M, Sedighim S, Palomino K, Conlisk D, Momper JD, de Guglielmo G, George O (2020) Oxycodone self-administration and withdrawal behaviors in male and female Wistar rats. Psychopharmacology (Berl) 237:1545-1555.

Klepstad P, Fladvad T, Skorpen F, Bjordal K, Caraceni A, Dale O, Davies A, Kloke M, Lundström S, Maltoni M, Radbruch L, Sabatowski R, Sigurdardottir V, Strasser F, Fayers PM, Kaasa S (2011) Influence from genetic variability on opioid use for cancer pain: a European genetic association study of 2294 cancer pain patients. Pain 152:1139-1145.

Kranzler HR, Zhou H, Kember RL, Smith RV, Justice AC, Damrauer S, Tsao PS, Klarin D, Baras A, Reid J, Overton J, Rader DJ, Cheng Z, Tate JP, Becker WC, Concato J, Xu K, Polimanti R, Zhao H, Gelernter J (2019) Genome-wide association study of alcohol consumption and use disorder in 274,424 individuals from multiple populations. Nat Commun 10:1499.

Kreek MJ, Nielsen DA, Butelman ER, LaForge KS (2005) Genetic influences on impulsivity, risk taking, stress responsivity and vulnerability to drug abuse and addiction. Nat Neurosci 8:1450-1457.

Lenoir M, Augier E, Vouillac C, Ahmed SH (2013) A Choice-based screening method for compulsive drug users in rats. Curr Protoc Neurosci 1:1-17.

Liu M, Jiang Y, Wedow R, Li Y, Brazel DM, Chen F, Datta G, DavilaVelderrain J, McGuire D, Tian C, Zhan X; 23andMe Research Team; HUNT All-In Psychiatry, Choquet H, Docherty AR, Faul JD, Foerster JR, Fritsche LG, Gabrielsen ME, Gordon SD, et al. (2019) Association studies of up to 1.2 million individuals yield new 
insights into the genetic etiology of tobacco and alcohol use. Nat Genet 51:237-244.

Paxinos G, Watson C (2004) The Rat Brain in Stereotaxic Coordinates - The New Coronal Set, Ed 5. San Diego:Academic Press.

Polimanti R, Walters RK, Johnson EC, McClintick JN, Adkins AE, Adkins DE, Bacanu SA, Bierut LJ, Bigdeli TB, Brown S, Bucholz KK, Copeland WE, Costello EJ, Degenhardt L, Farrer LA, Foroud TM, Fox L, Goate AM, Grucza R, Hack LM, et al. (2020) Leveraging genome-wide data to investigate differences between opioid use vs. opioid dependence in 41,176 individuals from the Psychiatric Genomics Consortium. Mol Psychiatry 25:1673-1687.

Ramôa CP, Doyle SE, Naim DW, Lynch WJ (2013) Estradiol as a mechanism for sex differences in the development of an addicted phenotype following extended access cocaine self-administration. Neuropsychopharmacology 38:1698-1705.

Rasooly RS, Gossett DR, Henderson MK, Hubel A, Thibodeau SN (2017) High-Throughput Processing to Preserve Viable Cells: A Precision Medicine Initiative Cohort Program Workshop. Biopreserv Biobank 15:341-343.

Roth ME, Carroll ME (2004) Sex differences in the escalation of intravenous cocaine intake following long- or short-access to cocaine self-administration. Pharmacol Biochem Behav 78:199-207.

Sanchez-Roige S, Palmer AA, Fontanillas P, Elson SL; 23andMe Research Team, the Substance Use Disorder Working Group of the Psychiatric Genomics Consortium, Adams MJ, Howard DM, Edenberg HJ, Davies G, Crist RC, Deary IJ, Mclntosh AM, Clarke TK (2019a) Genome-wide association study meta-analysis of the alcohol use disorders identification test (AUDIT) in two populationbased cohorts. Am J Psychiatry 176:107-118.

Sanchez-Roige S, Fontanillas P, Elson SL, Gray JC, de Wit H, Davis LK, MacKillop J, Palmer AA (2019b) Genome-wide association study of alcohol use disorder identification test (AUDIT) scores in 20328 research participants of European ancestry. Addict Biol 24:121-131.

Sanchez-Roige S, Palmer AA, Clarke TK (2020) Recent efforts to dissect the genetic basis of alcohol use and abuse. Biol Psychiatry 87:609-618.

Sloan Z, Arends D, W. Broman K, Centeno A, Furlotte N, Nijveen H, Yan L, Zhou X, W. Williams R, Prins P (2016) GeneNetwork: framework for web-based genetics. J Open Source Softw 1:25.

Smith JR, Hayman GT, Wang SJ, Laulederkind SJF, Hoffman MJ, Kaldunski ML, Tutaj M, Thota J, Nalabolu HS, Ellanki SLR, Tutaj MA, De Pons JL, Kwitek AE, Dwinell MR, Shimoyama ME (2020) The year of the rat: the rat genome database at 20: a multi-species knowledgebase and analysis platform. Nucleic Acids Res 48: D731-D742.

Solberg Woods LC, Palmer AA (2019) Using heterogeneous stocks for fine-mapping genetically complex traits. Methods Mol Biol 2018:233-247.

STAR Consortium, Saar K, Beck A, Bihoreau MT, Birney E, Brocklebank D, Chen Y, Cuppen E, Demonchy S, Dopazo J, Flicek P, Foglio M, Fujiyama A, Gut IG, Gauguier D, Guigo R, Guryev V,
Heinig M, Hummel O, Jahn N, et al. (2008) SNP and haplotype mapping for genetic analysis in the rat. Nat Genet 40:560-566.

Takahashi K, Yamanaka S (2006) Induction of pluripotent stem cells from mouse embryonic and adult fibroblast cultures by defined factors. Cell 126:663-676.

Vadivelu N, Kai AM, Kodumudi V, Sramcik J, Kaye AD (2018) The opioid crisis: a comprehensive overview. Curr Pain Headache Rep 22:16.

Valdar W, Solberg LC, Gauguier D, Burnett S, Klenerman P, Cookson WO, Taylor MS, Rawlins JNP, Mott R, Flint J (2006) Genome-wide genetic association of complex traits in heterogeneous stock mice. Nat Genet 38:879-887.

Vanderschuren LJMJ, Everitt BJ (2004) Drug seeking becomes compulsive after prolonged cocaine self-administration. Science 305:1017-1019.

Vendruscolo LF, Schlosburg JE, Misra KK, Chen SA, Greenwell TN, Koob GF (2011) Escalation patterns of varying periods of heroin access. Pharmacol Biochem Behav 98:570-574.

Vowles KE, McEntee ML, Julnes PS, Frohe T, Ney JP, van der Goes DN (2015) Rates of opioid misuse, abuse, and addiction in chronic pain: a systematic review and data synthesis. Pain 156 156:569576.

Wade CL, Vendruscolo LF, Schlosburg JE, Hernandez DO, Koob GF (2015) Compulsive-like responding for opioid analgesics in rats with extended access. Neuropsychopharmacology 40:421-428.

Walters RK, Polimanti R, Johnson EC, McClintick JN, Adams MJ, Adkins AE, Aliev F, Bacanu SA, Batzler A, Bertelsen S, Biernacka JM, Bigdeli TB, Chen LS, Clarke TK, Chou YL, Degenhardt F, Docherty AR, Edwards AC, Fontanillas P, Foo JC, et al. (2018) Transancestral GWAS of alcohol dependence reveals common genetic underpinnings with psychiatric disorders. Nat Neurosci 21:1656-1669.

Wee S, Mandyam CD, Lekic DM, Koob GF (2008) $\alpha 1$-Noradrenergic system role in increased motivation for cocaine intake in rats with prolonged access. Eur Neuropsychopharmacol 18:303-311.

White SR, Amarante LM, Kravitz AV, Laubach M (2019) The future is open: open-source tools for behavioral neuroscience research. eNeuro 6:ENEURO.0223-19.2019-5.

Xue Y, Steketee JD, Sun W (2012) Inactivation of the central nucleus of the amygdala reduces the effect of punishment on cocaine selfadministration in rats. Eur J Neurosci 35:775-783.

Zhou H, Sealock JM, Sanchez-Roige S, Clarke TK, Levey DF, Cheng Z, Li B, Polimanti R, Kember RL, Smith RV, Thygesen JH, Morgan MY, Atkinson SR, Thursz MR, Nyegaard M, Mattheisen $M$, Børglum AD, Johnson EC, Justice AC, Palmer AA, et al. (2020a) Genome-wide meta-analysis of problematic alcohol use in 435,563 individuals yields insights into biology and relationships with other traits. Nat Neurosci 23:809-818.

Zhou H, Rentsch CT, Cheng Z, Kember RL, Nunez YZ, Sherva RM, Tate JP, Dao C, Xu K, Polimanti R, Farrer LA, Justice AC, Kranzler HR, Gelernter J; Veterans Affairs Million Veteran Program (2020b) Association of OPRM1 functional coding variant with opioid use disorder: a genome-wide association study. JAMA Psychiatry 77:1072-1080. 